\section{Cerebral blood flow and cerebrovascular reactivity in Parkinson's disease: Relationship with severity of motor symptoms}

\section{Laura Pelizzari, ${ }^{1}$ \\ Maria Marcella Laganà, ${ }^{1}$ \\ Federica Rossetto, ${ }^{1}$ Niels Bergsland, ${ }^{1,2}$ \\ Giuseppe Baselli, ${ }^{3}$ Mario Clerici, ${ }^{1,4}$ \\ Raffaello Nemni, ${ }^{1,4}$ Mario Meloni, ${ }^{1}$ \\ Francesca Baglio ${ }^{1}$}

${ }^{1}$ IRCCS, Fondazione Don Carlo

Gnocchi, Milano, Italy; ${ }^{2}$ Buffalo

Neuroimaging Analysis Center,

Department of Neurology, Jacobs School

of Medicine and Biomedical Sciences,

University at Buffalo, State University of

New York, Buffalo, NY, USA;

${ }^{3}$ Department of Electronics, Information and Bioengineering, Politecnico di

Milano, Milano, Italy; ${ }^{4}$ Department of Physiopathology and Transplants,

University of Milano, Milano, Italy

\section{Background}

Parkinson's disease (PD) is a progressive neurodegenerative disorder that mainly affects motor function. ${ }^{1}$ Neurovascular unit (NVU) disruption has been hypothesized to play a role in neurodegenerative mechanisms in PD. ${ }^{2}$ However, its relationship with the development and progression of the disease is still unclear. ${ }^{2}$ The aims of this study were to assess cerebral blood flow (CBF) and cerebrovascular reactivity (CVR) in mild to moderate stages of PD, and their correlations with the severity of motor symptoms.

\section{Methods}

Arterial spin labeling (ASL) MRI was acquired for twenty-eight $\mathrm{PD}$ patients (66.6 \pm 8.6 years, 22 males, median [IQR] $\mathrm{H} \& \mathrm{Y}=1.5 \quad[1-1.9]$, median [IQR] $\mathrm{MoCA}=22.9$ [20-24.9]) and thirty-two ageand sex-matched healthy controls (HC, 63.1 \pm 8.1 years, 20 males) to derive $\mathrm{CBF}$ maps. A subgroup of thirteen PD patients and thirteen $\mathrm{HC}$ was also scanned at hypercapnia to compute CVR. Median CBF and CVR were computed within cortical and subcortical regions of the motor network (precentral gyrus, postcentral gyrus, supplementary motor area, striatum, pallidum, thalamus, red nucleus, substantia nigra and subthalamic nucleus). PD-vs-HC differences were tested for both $\mathrm{CBF}$ and CVR, and the correlation between them and the severity of PD motor symptoms (quantified with UPDRS III) was assessed. Multiple comparisons were corrected using the false discovery rate (FDR) method.

\section{Results}

No significant CBF/CVR differences were found between PD and HC. Positive significant $\left(\mathrm{p}_{\mathrm{FDR}}<0.05\right)$ CBF-UPDRS III correlation was observed in the precentral gyrus $\left(\mathrm{rho}=0.633, \mathrm{p}_{\mathrm{FDR}}=0.001\right)$, postcentral gyrus (rho $=0.673, \mathrm{p}_{\mathrm{FDR}}<0.001$ ), supplementary motor area $(\mathrm{rho}=0.543, \mathrm{pFDR}=0.003)$, corpus striatum $\left(\mathrm{rho}=0.493, \mathrm{p}_{\mathrm{FDR}}=0.015\right)$, pallidum ( $\left.\mathrm{rho}=0.447, \mathrm{p}_{\mathrm{FDR}}=0.023\right)$, thalamus (rho=0.648, $\mathrm{p}_{\mathrm{FDR}}=0.002$ ), red nucleus (rho=0.555, $\left.\mathrm{p}_{\mathrm{FDR}}=0.009\right)$ and substantia nigra (rho $\left.=0.485, \mathrm{p}_{\mathrm{FDR}}=0.015\right)$. Significant negative CVR-UPDRS III correlation was found in the striatum $(\mathrm{rho}=-0.720$, $\left.\mathrm{p}_{\mathrm{FDR}}=0.048\right)$.
Correspondence: Laura Pelizzari, IRCCS, Fondazione Don Carlo Gnocchi, Milano, Italy.

E-mail: lapelizzari@dongnocchi.it

Key words: Neurovascular diseases; meeting.

Conference presentation: $9^{\text {th }}$ Annual Meeting of the International Society of Neurovascular Disease (ISNVD), May $30^{\text {th }}-31^{\text {st }}, 2019$, Ferrara, Italy.

Award: Young Investigator Award Session Third prize.

This work is licensed under a Creative Commons Attribution 4.0 License (by-nc 4.0).

(C) Copyright: the Author(s), 2019

Licensee PAGEPress, Italy

Veins and Lymphatics 2019; 8:8442

doi:10.4081/vl.2019.8442

\section{Conclusions}

These results suggest that there might be a neurovascular involvement in the motor network at the mild to moderate stages of PD. CBF and CVR assessment provides indirect non-invasive information about NVU integrity and may be used as imaging markers for PD longitudinal evaluation.

\section{References}

1. Obeso, JA, Stamelou M, Goetz, CG, et al. Past, present, and future of Parkinson's disease: a special essay on the 200th anniversary of the shaking palsy. Mov Disord 2017;32:1264-310.

2. Sweeney MD, Sagare AP, Zlokovic BV. Blood-brain barrier breakdown in Alzheimer disease and other neurodegenerative disorders. Nat Rev Neurol 2018;14:133. 